\title{
Aumento de la productividad de plantaciones de eucalipto en la IX Región a través de técnicas racionales de manejo del sistema suelo-agua-planta*
}

\author{
Increase of productivity of eucalypts by means of rational technology applied \\ to the soil-plant-water system \\ EMILIO GUERRA ${ }^{1}$, DOMINGO SUAREZ ${ }^{2}$ \\ ${ }^{1}$ Facultad de Ciencias Agropecuarias y Forestales, Universidad Católica de Temuco, \\ Casilla 15-D. Temuco, Chile. \\ ${ }^{2}$ Facultad de Agronomía e Ingeniería Forestal, \\ Pontificia Universidad Católica de Chile, Casilla $306 \mathrm{~N}^{\circ} 22$, Santiago, Chile.
}

\begin{abstract}
SUMMARY
The principal objective of this project is to obtain a $15 \%$ production increase in large or small eucalyptus plantations. It involves adopting an agronomic approach to the forestry field. considering the eucalyptus trees as plants rather than as forests. Thus, increasing raw material for the cellulose industry as well as the one destined for the international chip markets. The project was developed by the Universidad Católica de Temuco and Pontificia Universidad Católica de Chile, with the cooperation of Instituto Forestal in the technological transference area, and is funded by the Fondo de Fomento al Desarrollo Científico y Tecnológico (FONDEF), Forestal Mininco S.A., Forestal y Agrícola Monte Aguila S.A., Bosques Cautín S.A. and ANAGRA S.A.
\end{abstract}

Key words: Eucalypts, productivity, technology.

\section{RESUMEN}

El presente proyecto tiene como objetivo principal aumentar la productividad de las plantaciones de eucalipto en un $15 \%$ en términos de biomasa, ya sea se trate de grandes empresas o pequeños y medianos propietarios. Este hecho implicaría que mediante la incorporación de la tecnología a desarrollar por el proyecto se podrá contar con mayor materia prima para la industria de la celulosa y del mercado internacional de las astillas, optimizando el recurso suelo, agua y planta y reordenando la utilización de otros recursos asociados que permiten dicho fin.

La principal característica de éste es que incorpora técnicas agrícolas a la actividad forestal. La forma de integración de estas dos disciplinas viene a confirmar la necesidad de tratar a las plantaciones forestales como un cultivo que requiere una intensa dedicación desde su establecimiento, a favor de lograr mejores rendimientos.

Este proyecto es desarrollado actualmente en conjunto por investigadores y técnicos de la Universidad Católica de Temuco y Pontificia Universidad Católica de Chile, cuenta con el apoyo del Instituto Forestal para la transferencia tecnológica y es financiado por el Fondo de Fomento al Desarrollo Científico y Tecnológico (FONDEF), Forestal Mininco S.A., Forestal y Agrícola Monte Aguila S.A., Bosques Cautín S.A. y ANAGRA S.A.

Palabras claves: Eucalyptus, productividad, tecnología.

\section{FUNDAMENTOS DEL PROYECTO}

Para lograr el aumento de la productividad se debe considerar que gran parte de la investigación realizada en eucalipto en Chile ha estado centrada

Proyecto FONDEF D97 I 2009 en determinar la respuesta a técnicas silvícolas como la fertilización, control de malezas, control del espaciamiento y preparación del suelo, entre otras, en diferentes tipos de ecosistemas. Adicionalmente, las tasas de crecimiento y la producción de madera han sido evaluadas en períodos muy cortos de tiempo. Este tipo de investigación proporciona en la actualidad la base de la información 
empírica para la toma de decisiones de manejo de los rodales. Sin embargo, los resultados de este tipo de experimentación no pueden ser fácilmente extrapolados a otros sitios o condiciones climáticas o ser utilizados para explorar las consecuencias de las decisiones de manejo fuera del rango donde se hizo tal experimentación.

A partir de este argumento, es que se hace necesario entender y cuantificar el efecto de los factores que determinan el crecimiento de los árboles y estudiarlos bajo un amplio rango de condiciones, que permitan desarrollar modelos para predecir las posibles respuestas de las técnicas de manejo estudiadas a las diferentes condiciones edafoclimáticas sobre las cuales están establecidas las plantaciones. Para ello se requiere focalizar la investigación en las relaciones que existen entre estas variables al nivel de los procesos fisiológicos de las plantas.

Además, si se sabe que las tasas de crecimiento de la demanda por celulosa se están recuperando a sus niveles históricos del $2.5 \%$ al $3.0 \%$ anual, que los proyectos industriales que se están desarrollando en este momento en el mundo van a aumentar la capacidad de producción en un $1.8 \%$ y que se están implementando interesantes proyectos celulósicos en la VI, VIII, IX y X regiones, es lógico esperar que la tecnología a desarrollar por el proyecto se extienda rápidamente.

En este contexto, las empresas asociadas al proyecto han aportado su experiencia en cuanto al manejo y tratamiento de las plantaciones y, por otra parte, las Universidades han entregado un invaluable aporte científico-tecnológico al proyecto, el cual es financiado, junto a las empresas, por el fondo de Fomento al Desarrollo Científico y Tecnológico (FONDEF).

\section{OBJETIVOS}

Los objetivos que persigue el proyecto tienen relación con el aumento de la productividad de las plantaciones de eucalipto, Eucalyptus globulus y Eucalyptus nitens, de la IX Región, mediante la adopción de criterios racionales de manejo, asociados al establecimiento de plantaciones en relación al sistema suelo-agua-planta. Además, se busca incrementar los conocimientos de técnicas de establecimiento de eucalipto que promuevan una mayor sobrevivencia de las plántulas y una mayor tasa de crecimiento y uniformidad de la planta- ción, y que proyectados a la cosecha se traduzcan en la obtención de una mayor cantidad de volumen de madera pulpable.

Por último, se pretende generar un adecuado programa de transferencia para incorporar las nuevas tecnologías de manejo de establecimiento a las plantaciones de eucalipto en la IX Región y en el país.

\section{PARTICIPANTES}

En el desarrollo de la presente iniciativa participa un destacado grupo de investigadores y técnicos de la Universidad Católica de Temuco y Pontificia Universidad Católica de Chile, convocando el valioso aporte de las siguientes empresas e instituciones que conforman las contrapartes: Forestal y Agrícola Monte Aguila S.A., Forestal Mininco S.A., Bosques Cautín S.A., ANAGRA S.A. e Instituto Forestal.

\section{ESTRUCTURA DEL PROYECTO}

En términos operativos, la Universidad Católica de Temuco es la encargada de gestionar y coordinar el proyecto, en conjunto con la Pontificia Universidad Católica de Chile, siendo la Universidad Católica de Temuco el nexo entre las instituciones y empresas participantes. Este proyecto tiene una duración de tres años, comenzó a operar en abril de 1998 y finalizará en abril de 2001.

El proyecto considera las siguientes áreas de estudio:

- Estudio de tasa de crecimiento y absorción de nutrientes bajo condiciones de secano y riego.

- Estudio de la tasa de crecimiento y absorción de nutrientes bajo diferentes niveles de densidad de plantación.

- Estudio de diferentes nutrientes y formas de aplicación.

- Estudio de diferentes sistemas de preparación de suelo y control de malezas.

- Estudio de época de plantación en diferentes ecosistemas.

- Estudio del efecto de la calidad de la planta.

- Estudio de fertilización en rebrote.

- Estudio de riego y fertilización.

Los estudios antes mencionados se están desarrollando en los predios seleccionados por las 
empresas asociadas al proyecto, en los cuales se han instalado los distintos ensayos considerando seis ecosistemas: Secano Costero Norte (Colcura), Secano Costero Sur (Cañete), Valle Central Norte (Mulchén), Valle Central Sur (Los Sauces), Precordillera Norte (Huepil) y Precordillera Sur (Vilcún), todos en el sur de la VIII y norte de la IX Región.

Un aspecto importante considerado dentro del proyecto corresponde a la transferencia tecnológica, la que ha sido asignada al Instituto Forestal, de tal modo que se defina una estrategia de transferencia, los mecanismos para viabilizar la estrategia y los canales de difusión. De acuerdo a lo anterior, un programa de transferencia será dirigido a profesionales y técnicos de empresas así como de organismos públicos y privados asociados a la producción forestal; por otra parte, se pretende llegar también a pequeños y medianos propietarios a través de profesionales de empresas como de organismos públicos que operan en la transferencia tecnológica vinculada a la producción silvoagropecuaria.

Los mecanismos a utilizar se basan en un contacto y transmisión directa de los avances y resultados de la investigación con los usuarios.

Se pretende definir distintos medios de difusión de los avances del proyecto, entre los cuales se pueden mencionar invitaciones directas, publicaciones en periódicos e información a través de internet. Se considera además la realización de publicaciones científicas y de divulgación para presentar en revistas sectoriales o científicas a nivel nacional e internacional.

\section{CONTRIBUCIONES DEL PROYECTO}

Las contribuciones del proyecto son de dos tipos: resultados verificables durante el período de vigencia del financiamiento FONDEF, e impactos que se apreciarán en el mediano y largo plazo. Entre los primeros, en el corto plazo, se considera:

- Elaboración de material audiovisual de carácter divulgativo, como notas técnicas, folletos, compendios, protocolos de cultivo o manuales operativos de establecimiento y manejo y videos.
Por su parte, los impactos que se esperan de la aplicación de los resultados del proyecto se pueden resumir en:

- Impactos científico-tecnológicos:

- Lograr una mayor comprensión de la ecofisiología del eucalipto.

- Introducir métodos de calificación de sitios que complementen a los ya existentes.

- Aportes en relación al uso y aplicación de fertilizantes:

- Desarrollo de criterios y técnicas para un control más eficiente en términos económicos y ecológicos.

- Iniciar actividades y equipamiento para establecer trabajos de largo aliento.

- Información dasométrica.

- Fortalecimiento del conocimiento y experiencia práctica de los equipos de trabajo.

- Impactos económico-sociales:

- Aumento de la productividad del cultivo.

- Disminución de costos por optimización de procesos.

- Aumento de las ventajas competitivas.

- Generación de oportunidades de reconversión agrícola.

- Aumento del valor del suelo.

- Generación de polos de desarrollo en pueblos rurales.

- Impactos ambientales:

- Disminución de la erosión.

- Aprovechamiento del recurso suelo.

- Absorción de $\mathrm{CO}_{2}$.

- Impactos institucionales:

- Consolidación equipos de trabajo.

- Estimulación del trabajo interinstitucional.

- Traspaso de conocimientos entre equipos.

- Consolidación de la relación universidadempresa.

- Impulso al desarrollo de tesis de grado. 